\title{
The Changes of CTX, DPD, Osteocalcin, and Bone Mineral Density During the Postmenopausal Period
}

\author{
Seok Gyo Park, MD, Seong Uk Jeong, MD, Jae Hyun Lee, MD, Sang Hyeong Ryu, MD, \\ Ho Joong Jeong, MD, PhD, Young Joo Sim, MD, PhD, Dong Kyu Kim, MD, Ghi Chan Kim, MD, PhD \\ Department of Physical Medicine and Rehabilitation, Kosin University College of Medicine, Busan, Korea
}

\begin{abstract}
Objective To investigate appropriate treatment time and useful bone turnover markers (BTMs) for monitoring bone turnover during the postmenopausal period, we analyzed changes of two bone resorption markers; serum carboxyterminal telopeptide of collagen I (s-CTX), urine deoxypyridinoline (u-DPD), one bone formation marker; serum osteocalcin (s-OC), and bone mineral density (BMD) in Korean postmenopausal women.

Methods Seventy-eight menopausal women were divided into three groups according to postmenopausal period: group I ( $0-5$ years), group II (6-10 years), group III ( $\geq 10$ years). All groups were subdivided into an osteoporosis group (T-score $\leq-2.5$ ) and a non-osteoporosis group (T-score >-2.5). BTMs such as s-CTX, u-DPD, s-OC, and BMD $\left(\mathrm{g} / \mathrm{cm}^{2}\right)$ were measured by dual-energy X-ray absorptiometry (DXA) in all patients. Analysis of variables among groups based on the postmenopausal period was performed using ANOVA.

Results There was significant negative correlation between BMD and postmenopausal period. The levels of all BTMs including s-CTX, u-DPD, and s-OC were highest in group II and the increased levels of all BTMs subsequently declined in group III. The levels of BTMs were higher in the osteoporosis groups than in the nonosteoporosis groups in all subjects. It was statistically significant that the level of s-CTX in group I was higher in the osteoporosis group than in the non-osteoporosis group.

Conclusion This study showed that bone resorption and bone formation were the highest 5-10 years after menopause, and s-CTX is more useful than u-DPD among the bone resorption markers. It's important to measure serially both BMD and BTM within 10 years after menopause for accurate diagnosis and management for postmenopausal osteoporosis.
\end{abstract}

Keywords Postmenopause, Osteoporosis, Biomarker, Bone density

Received June 25, 2017; Accepted August 25, 2017

Corresponding author: Ghi Chan Kim

Department of Physical Medicine and Rehabilitation, Kosin University College of Medicine, 262 Gamcheon-ro, Seo-gu, Busan 49267, Korea. Tel: +8251-990-6261, Fax: +82-51-241-2019, E-mail: ghichan@hotmail.com

ORCID: Seok Gyo Park (http://orcid.org/0000-0002-5809-3313); Seong Uk Jeong (http://orcid.org/0000-0002-1267-5879); Jae Hyun Lee (http://orcid. org/0000-0002-4927-9722); Sang Hyeong Ryu (http://orcid.org/0000-0002-9233-2525); Ho Joong Jeong (http://orcid.org/0000-0002-0607-2799); Young Joo Sim (http://orcid.org/0000-0002-0640-8766); Dong Kyu Kim (http://orcid.org/0000-0002-9484-6562); Ghi Chan Kim (http://orcid.org/0000-00024954-3175)

(c) This is an open-access article distributed under the terms of the Creative Commons Attribution Non-Commercial License (http://creativecommons.org/ licenses/by-nc/4.0) which permits unrestricted noncommercial use, distribution, and reproduction in any medium, provided the original work is properly cited. Copyright $\odot 2018$ by Korean Academy of Rehabilitation Medicine 


\section{INTRODUCTION}

Osteoporosis is a metabolic disease that changes bone strength and homogeneity because of a transition in bone structure and is associated with increased risk of fracture $[1,2]$. Primary osteoporosis occurs in people who do not have endocrinopathy or other diseases that explain the change in bone mass. Postmenopausal osteoporosis results from increased bone resorption and calcium levels, decreased levels of parathyroid hormone, and absorption of intestinal calcium in the postmenopausal period. Secondary osteoporosis is the result of certain medical conditions, diseases, or drugs that interfere with achieving peak bone mass or make it easier for a person to have accelerated bone loss. These include endocrine, nutritional, and hereditary factors, as well as focal osteoporosis and immobilization osteoporosis, which that can occur after stroke or spinal cord injury $[3,4]$. The prevalence of osteoporosis is higher in women than men because of increased bone resorption that results from decreasing estrogen secretion during menopause [5-7].

In postmenopausal women, acceleration of bone resorption begins at about $\mathbf{2 \%}-\mathbf{3 \%}$ of bone resorption every year immediately after menopause, and the bone mass decreases exponentially [8]. To prevent and treat osteoporosis and the fractures it causes, it is important to identify the most active period of bone resorption and the high risk of osteoporosis.

Dual-energy X-ray absorptiometry (DXA) is commonly used to measure bone mineral density (BMD) to assess the risk of fracture in postmenopausal osteoporosis [9]. BMD measured by DXA is a useful way to quantify the risk of fracture, but the bone strength associated with fracture risk depends not only on bone density, but also on such factors of the skeleton as size, shape, and steric structure.

Bone turnover markers (BTMs) have recently been used to evaluate bone turnover rate and to monitor therapeutic response to osteoporosis [10-12]. BTMs provide dynamic information about skeletal status, independently or sometimes complementarily to BMD measurement. It is reported that high bone resorption markers with low $\mathrm{BMD}$ in the femur are more likely to predict fractures than those with only low BMD [13], and recent studies have shown that increased levels of BTMs are associated with an increased risk of osteoporotic fracture indepen- dently from BMD [14]. Therefore, BMD and BTMs should be measured together to assess risk of fracture more accurately.

Postmenopausal estrogen deficiency, in which the increase in osteoclastic resorption is not accompanied by a corresponding increase in osteoblastic activity, ultimately leads to more reabsorption than bone accumulation and cause a net loss of the bone, resulting in damage to the normal cycle of bone remodeling [15]. Markers commonly used as bone formation marker include serum osteocalcin (s-OC), serum bone-specific alkaline phosphatase, and carboxyterminal propeptide of type 1 procollagen, whereas markers frequently used as bone resorption include serum carboxyterminal telopeptide of collagen I (s-CTX), urine deoxypyridinoline (u-DPD), free pyridinolines, free deoxypyridinoline, and urine amino terminal crosslinked telopeptides of collagen I [16]. In clinical practice, s-OC is the most commonly used bone formation marker, whereas s-CTX or u-DPD are the most commonly used bone resorption markers because of their convenience and sensitivity.

In this study, we analyzed differences in BMD and BTMs according to postmenopausal period and evaluated the correlations between BMD and BTMs that can be useful for managing postmenopausal osteoporosis.

\section{MATERIALS AND METHODS}

\section{Subjects}

A retrospective investigation was conducted using the medical records of 2,068 women who had undergone both BMD and BTMs (s-OC, s-CTX, u-DPD) measurement at Kosin University Gospel Hospital from January 2006 to October 2015 (IRB No. 201603-BM-005). Of these, 867 subjects with an interval between exams of less than 1 week were included; 798 women were excluded because the time of menopause was not clear, they had a history of metabolic disease or fracture, or had a history of medications that can affect bone metabolism or cause renal failure. The remaining 78 subjects were included in the study. Among the women who visited the hospital, menopause was defined as a serum FSH level (follicular fluid) of $50 \mathrm{mIU} / \mathrm{mL}$ and absence of menstruation for more than 1 year. The 78 subjects were divided into three groups: group I, menopause less than 5 years; group II, menopause over 5 years and less than 10 years; group 
III, menopause more than 10 years. Each group was then subdivided into an osteoporosis group and non-osteoporosis group based on BMD.

\section{Assessment}

BMD was measured in six lumbar areas (L1-2, L1-3, L1-4, L2-3, L2-4, L3-4) and two femur areas (femur neck, femur trochanter) by DXA (Lunar Radiation Corp., Madison, WI, USA). To diagnose osteoporosis, the T-score obtained by dividing the mean bone density of a young adult group of the same gender (reported by Lunar) was used as the standard, and a T-score at or below -2.5 was defined as osteoporosis. For each of the major groups (I, II, and III), patients were assigned to an osteoporosis subgroup if any one of six lumbar areas or two femur areas had a T-score at or below -2.5 or to a non-osteoporosis group if not.

To examine quantitatively the changes in BMD based on postmenopausal period, BMD of each area (in $\mathrm{g} / \mathrm{cm}^{2}$ ) was compared between groups. To examine the changes in BTMs according to postmenopausal period, the means of groups were compared, and BTM levels were also compared between the osteoporosis and non-osteoporosis groups within each group.

To reduce biological variability, BTM sampling was performed at a specific time (early in the morning after an overnight fast).

\section{Statistical analysis}

All data in each group are presented as mean \pm standard deviation, and statistical analysis was performed using Statistical Package for the Social Sciences (SPSS) for Windows Release 22.0 (IBM, Armonk, NY, USA). Comparison of baseline characteristics between groups was assessed by Student t-tests. Comparisons of BTMs between osteoporosis and non-osteoporosis in each group were ana- lyzed in the same manner. Analysis of variables between groups based on postmenopausal period was performed using ANOVA with $\mathrm{p}<0.05$ considered statistically significant.

\section{RESULTS}

\section{Demographic characteristics of the subjects}

Of the 78 subjects, 35, 21, and 22 individuals were assigned to groups I, II, and III, respectively, and the mean postmenopausal period was $2.3 \pm 1.2,8.0 \pm 1.1$, and $15.9 \pm 1.6$ years, respectively. The mean age of each group was $52.3 \pm 3.4,57.5 \pm 4.3$, and $64.4 \pm 5.7$ years, and the mean age of the menopausal group was $50.0 \pm 3.3,49.5 \pm 4.2$, and $48.5 \pm 4.4$ years, respectively (Table 1 ). The mean age of menopausal women was not statistically significant, but the age of menopause tended to be lower in the elderly.

\section{Bone mineral density according to postmenopausal period}

BMD in six areas of the lumbar (L1-2, L1-3, L1-4, L2-3, L2-4, L3-4), femur neck, and femur trochanter decreased significantly as postmenopausal period increased $(p<0.05)$. In particular, the difference in BMD between groups II and III was greater than that between groups I and II (Table 2).

\section{Changes in BTM according to postmenopausal period}

Levels of s-OC, s-CTX, and u-DPD all increased more in group II than in group I, but decreased in group III compared to Group II. In particular, for s-CTX and s-OC, levels were highest in group II $(0.53 \pm 0.55 \mathrm{ng} / \mathrm{mL}$ and $16.2 \pm 13.2 \mathrm{ng} / \mathrm{mL}$, respectively) and the lowest in group III $(0.28 \pm 0.22 \mathrm{ng} / \mathrm{mL}$ and $11.0 \pm 6.40 \mathrm{ng} / \mathrm{mL}$ respectively). The levels of $\mathrm{u}$-DPD were highest in group II (45.6 \pm 58.9 $\mathrm{nM})$, lower in group III $(30.4 \pm 29.4 \mathrm{nM})$, and lowest in

Table 1. Demographic characteristics of the study groups

\begin{tabular}{|lccc}
\hline & Group I & Group II & Group III \\
\hline Number of subjects & 35 & 21 & 22 \\
\hline Osteoporosis group & 14 & 7 & 13 \\
\hline Non-osteoporosis group & 21 & 14 & 9 \\
Age (yr) & $52.3 \pm 3.4$ & $57.5 \pm 4.3$ & $64.4 \pm 5.7$ \\
Menopausal age (yr) & $50.0 \pm 3.3$ & $49.5 \pm 4.2$ & $48.5 \pm 4.4$ \\
\hline Duration of menopause (yr) & $2.3 \pm 1.2$ & $8.0 \pm 1.1$ & $15.9 \pm 1.6$ \\
\hline
\end{tabular}

Values are presented as number or mean \pm standard deviation. 
Seok Gyo Park, et al.

Table 2. BMD according to postmenopausal period

\begin{tabular}{lcccc}
\hline & Group I & Group II & Group III & p-value \\
\hline L1-L2 & $1.06 \pm 0.14$ & $1.02 \pm 0.11$ & $0.96 \pm 0.12$ & $0.008^{*}$ \\
\hline L1-L3 & $1.10 \pm 0.14$ & $1.07 \pm 0.12$ & $1.00 \pm 0.12$ & $0.015^{*}$ \\
\hline L1-L4 & $1.13 \pm 0.14$ & $1.11 \pm 0.12$ & $1.03 \pm 0.14$ & $0.013^{*}$ \\
L2-L3 & $1.13 \pm 0.14$ & $1.10 \pm 0.13$ & $1.03 \pm 0.12$ & $0.016^{*}$ \\
L2-L4 & $1.16 \pm 0.15$ & $1.13 \pm 0.13$ & $1.06 \pm 0.14$ & $0.019^{*}$ \\
\hline L3-L4 & $1.19 \pm 0.15$ & $1.17 \pm 0.15$ & $1.09 \pm 0.16$ & $0.026^{*}$ \\
\hline Femur neck & $0.90 \pm 0.12$ & $0.88 \pm 0.09$ & $0.82 \pm 0.87$ & $0.025^{*}$ \\
Femur trochanter & $0.80 \pm 0.12$ & $0.75 \pm 0.13$ & $0.73 \pm 0.11$ & $0.048^{*}$ \\
\hline
\end{tabular}

Values are presented as mean \pm standard deviation.

BMD, bone mineral density; L, lumbar spine.

${ }^{*} \mathrm{p}<0.05$ by one-way analysis of variances among groups.

Table 3. BTM according to postmenopausal period

\begin{tabular}{lcccc}
\hline & Group I $(\mathbf{n}=\mathbf{3 5})$ & Group II $(\mathbf{n}=\mathbf{2 1})$ & Group III $(\mathbf{n}=\mathbf{2 2})$ & p-value \\
\hline $\mathrm{T}^{\mathrm{a})}$ & $\mathrm{a}$ & $\mathrm{b}$ & $\mathrm{a}$ & \\
$\mathrm{s}$-CTX & $0.30 \pm 0.18$ & $0.53 \pm 0.55$ & $0.21 \pm 0.15$ & $0.015^{*}$ \\
$\mathrm{u}$-DPD & $27.4 \pm 18.8$ & $49.7 \pm 63.6$ & $29.4 \pm 22.0$ & 0.133 \\
$\mathrm{~s}$-OC & $12.0 \pm 7.4$ & $15.7 \pm 12.9$ & $10.8 \pm 7.0$ & 0.291 \\
\hline
\end{tabular}

Values are presented as mean \pm standard deviation.

BTM, bone turnover marker; s-CTX, serum carboxy-terminal telopeptide of collagen I; u-DPD, urine deoxypyridinoline; s-OC, serum osteocalcin.

${ }^{\text {a) }}$ The same letters indicate non-significant difference between groups based on the Scheffe multiple comparison test. ${ }^{*} \mathrm{p}<0.05$ by one-way analysis of variances among groups.

group I $(25.8 \pm 17.0 \mathrm{nM})$. These tendencies among the groups were statistically significant for s-CTX only (Table 3, Fig. 1).

When groups were subdivided into osteoporosis and non-osteoporosis groups and the changes in levels of BTMs according to postmenopausal period were analyzed, identical results were observed. When we analyzed differences in BTMs between the osteoporosis groups and non-osteoporosis groups, s-CTX, u-DPD, and s-OC were all expressed at higher concentrations in the osteoporosis groups than in the non-osteoporosis groups; in particular, the level of s-CTX in group I and the level of s-OC in group II were significantly higher in the osteoporosis group than in the non-osteoporosis group (Table 4).

When the groups were evaluated according to postmenopausal period, levels of all BTMs were found to be highest between 7 and 8 years after menopause in the non-osteoporosis group and between 8 and 9 years after menopause in the osteoporosis group.

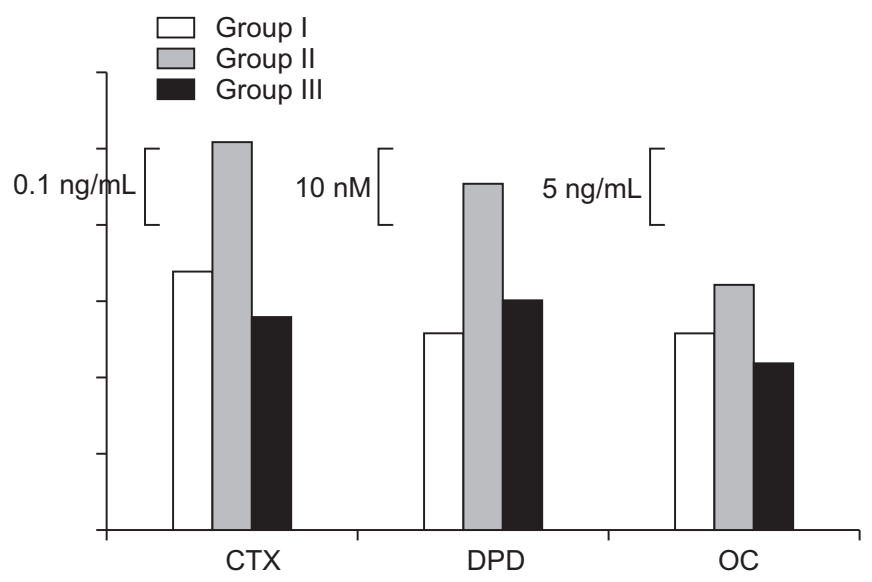

Fig. 1. Comparison of bone turnover markers among study groups. CTX, serum carboxy-terminal telopeptide of collagen I; DPD, urine deoxypyridinoline; OC, serum osteocalcin.

\section{DISCUSSION}

Bone density changes in two stages after birth, first reaching the peak bone mass in the early adult and grad- 
Table 4. Comparison of BTM between osteoporosis group and non-osteoporosis group

\begin{tabular}{llccc}
\hline & & Osteoporosis group & Non-osteoporosis group & p-value \\
\hline Group I & s-CTX & $0.44 \pm 0.20$ & $0.27 \pm 0.17$ & $0.035^{*}$ \\
& u-DPD & $29.0 \pm 22.4$ & $23.6 \pm 14.5$ & 0.389 \\
\hline \multirow{2}{*}{ Group II } & s-OC & $15.3 \pm 7.9$ & $11.3 \pm 7.9$ & 0.145 \\
& s-CTX & $0.75 \pm 0.76$ & $0.40 \pm 0.33$ & 0.173 \\
\hline \multirow{2}{*}{ Group III } & u-DPD & $64.0 \pm 34.7$ & $37.1 \pm 16.7$ & 0.371 \\
& s-OC & $23.8 \pm 17.7$ & $12.6 \pm 9.4$ & $0.048^{*}$ \\
& s-CTX & $0.30 \pm 0.26$ & $0.25 \pm 0.17$ & 0.559 \\
\hline
\end{tabular}

Values are presented as mean \pm standard deviation.

BTM, bone turnover marker; s-CTX, serum carboxy-terminal telopeptide of collagen I; u-DPD, urine deoxypyridinoline; s-OC, serum osteocalcin.

${ }^{*} \mathrm{p}<0.05$ by Student $\mathrm{t}$-test.

ually progressing to the bone-loss process. Peak bone mass is affected by several factors, including genetics, diet, physical activities, and hormone status. Bone loss occurs because of bone mass atrophy caused by hormone deficiency and aging, and postmenopausal osteoporosis is a typical example of bone loss because of hormone deficiency.

Postmenopausal osteoporosis is caused by estrogen deficiency and characterized by progress rapidly after menopause. This is associated with an increase in postmenopausal bone turnover and loss of the balance between bone resorption and bone formation, resulting in excessive bone resorption. Excessive bone loss is known to cause osteoporosis. Postmenopausal osteoporosis not only increases morbidity, but also decreases the quality of life of postmenopausal women. Therefore, prevention, early diagnosis, and early treatment of postmenopausal osteoporosis are important to reduce such problems.

BMD measurement by DXA has been commonly used to assess the risk of postmenopausal osteoporosis as well as senile osteoporosis, osteoporosis because of a variety of other causes, and associated fractures. However, BMD measurement using DXA had a limitation in that the three-dimensional bone structure was assessed by twodimensional images [17]. In another study, only half the fractures in women were identified by the WHO-defined osteoporosis criterion of a T-score $\leq-2.5$, and most of fractures were diagnosed as osteopenia $(-2.5<\mathrm{T}$-score $\leq-1)$ or as high BMD; this highlights the difficulty in assessing the risk of fracture by diagnosing osteoporosis using the ab- solute value of BMD only [18]. As an alternative, transiliac bone biopsy can be performed to examine the histological status of actual bone, but this procedure is invasive; new technologies that reflect the three-dimensional bone structure, such as quantitative computed tomography (QCT) or high-resolution QCT (HRQCT), are exciting alternatives.

Bone is a dynamic tissue that is continuously regenerated and destroyed. Recently, the importance of BTMs that indirectly reflect the process of bone remodeling has been increasing. In previous studies, the International Osteoporosis Foundation (IOF) reported that high-level BTMs were associated with increased risk of fracture in postmenopausal women independently from BMD. Many studies have also reported that measuring BMD and BTMs together is more helpful for predicting osteoporotic fractures than measuring BMD or BTMs alone [19-21]. Another study reported that subjects with a greater decrease in BTMs showed a greater decrease in osteoporotic fracture risk [22].

BTMs are enzymes that are released from osteoclasts or osteoblasts during the bone remodeling process or proteins released from the bone matrix. Serum or urine tests cab used to easily measure the bone turnover rate.

The purpose of this study was to analyze BMD and BTMs in postmenopausal women according to menopausal period in order to investigate changes of BMD and BTMs, to identify the significance of BTMs as well as BMD, and to decide on the most useful BTM for the diagnosis and evaluation of postmenopausal osteoporosis. 
BMD decreased significantly as the postmenopausal period increased, as is consistent with the results of previous studies [23]. In particular, BMD in group III decreased significantly. Because of age-related reduction of BMD in group III, the duration of the postmenopausal period in group III was much longer than that of the other groups, and the duration of bone loss because of estrogen deficiency was also long.

In this study, s-OC, s-CTX, and u-DPD increased from group I to group II, but decreased again in group III. This suggests that bone turnover occurs most actively between 5 and 10 years after menopause. When the groups were subdivided into osteoporosis and non-osteoporosis group, and the postmenopausal period was divided into intervals of 1 year, both groups had the highest BTM levels between 7 and 9 years after menopause. A more detailed analysis revealed that the three levels of BTMs in the non-osteoporotic groups showed the highest levels between 7 and 8 years after menopause, and the three levels of BTMs in the osteoporosis groups showed the highest levels between 8 and 9 years after menopause. This shows that the absolute amount of bone loss was greater and the duration of active bone loss was longer in the osteoporosis group. Approximately 10 years after menopause, bone turnover rate declines again. Therefore, early measurement of BTMs in postmenopausal women to identify high-risk osteoporosis and to administer antiresorptive sooner would be an effective for treatment of postmenopausal osteoporosis and reducing the risk of fracture.

In this study, a significant difference in s-CTX levels between the groups was observed, consistent with another study that reported a strong correlation between sCTX level and histologic features in bone biopsy [24]. The finding that s-CTX was significantly higher in the osteoporosis group than in the non-osteoporosis group, especially for group I, suggests that s-CTX is a better marker of bone resorption than $\mathrm{u}$-DPD during the early stage of postmenopausal period, when bone resorption begin to increase.

The bone resorption markers s-CTX and u-DPD showed similar trends over the postmenopausal period, but only the s-CTX showed statistically significant results, which could not be ruled out by the technical error of measuring BTMs. Because fasting and meals can affect BTM level, subjects should fast overnight and samples should be collected early in the morning. In this study, s-CTX could be collected at a set time, because it was a blood sample, but u-DPD was measured by allowing the patient to have a second urinalysis in the morning after fasting overnight. This potential bias might have resulted in the lack of significance of the u-DPD results.

When BTM levels were compared between the osteoporosis and non-osteoporosis groups in the main groups, we found that BTM levels were higher in the osteoporosis group than in the non-osteoporosis groups in all groups. In particular, the s-OC level of the osteoporosis group in group II was significantly higher than that of the nonosteoporosis group, probably because of the increase in bone formation caused by excessive bone loss in group II, which had the highest bone turnover rate. In group II, the difference in mean level of s-OC between the osteoporosis and non-osteoporosis groups was nearly twice that of other BTMs, thus giving it statistical significance.

Because only s-CTX among the three BTMs (s-CTX, uDPD, s-OC) was significantly different during the first 5 years after menopause, measurement of BTMs alone were limited in predicting postmenopausal osteoporosis. To accurately diagnose postmenopausal osteoporosis, BMD needs to be measured along with BTMs. Several studies reported that serial measurements of BTMs showed better correlations with BMD than single measurements of BTMs. Therefore, BTM should be measured serially with $\mathrm{BMD}$ to predict the early diagnosis of postmenopausal osteoporosis and the risk of osteoporotic fracture.

Bone-specific alkaline phosphatase (ALP), and procollagen type I N-terminal propeptide (PINP) have been reported to be the most sensitive markers among the bone formation markers, and further studies on these bone formation markers are also needed.

This study has some limitations. First, the sample size was small; hence, larger studies are needed to validate our findings. Second, patients were instructed to collect their second urine in the morning after overnight fasting for assessment of $\mathrm{u}$-DPD, so poor patient compliance might have affected sampling bias. Third, because the values of BTMs differ with renal function when measured from urine samples, urinary marker values are corrected for creatinine excretion, but assessment of creatinine excretion itself will have a measurement error and is affected by muscle quantity.

In conclusion, bone resorption and bone formation 
were the highest at 5-10 years after menopause. Compared to postmenopausal women without osteoporosis, women with postmenopausal osteoporosis have a higher bone turnover rate and longer duration of bone loss. Therefore, measuring BMD and BTMs regularly in the early postmenopausal period prior to the increase of the bone turnover rate is important for early diagnosis of postmenopausal osteoporosis and for deciding on the appropriate treatment time. Considering the convenience of testing and measurement error in a clinical setting, s-CTX is a more useful bone resorption marker than u-DPD. Serial measurement of BTMs and BMD within 5 years after menopause is essential to choose the best timing of treatment for postmenopausal osteoporosis. Starting antiresorptive therapy vigorously before 7-9 years after menopause, when bone turnover is the most active, may be an effective treatment for postmenopausal osteoporosis.

\section{CONFLICT OF INTEREST}

No potential conflict of interest relevant to this article was reported.

\section{REFERENCES}

1. Consensus development conference: diagnosis, prophylaxis, and treatment of osteoporosis. Am J Med 1993;94:646-50.

2. NIH Consensus Development Panel on Osteoporosis Prevention, Diagnosis, and Therapy. Osteoporosis prevention, diagnosis, and therapy. JAMA 2001;285: 785-95.

3. Kim G. Immobilization osteoporosis. Osteoporosis 2011;9:1-17.

4. Kim HD, Kim SH, Kim DK, Jeong HJ, Sim YJ, Kim GC. Change of bone mineral density and relationship to clinical parameters in male stroke patients. Ann Rehabil Med 2016;40:981-8.

5. Riggs BL, Melton LJ. Involutional osteoporosis. N Engl J Med 1986;314:1676-86.

6. Kim GC, Jeong HJ, Roh KH, Chung HC, Kim HY, Lee $\mathrm{HN}$. The changes of bone mineral density, biochemical bone markers, and lipid profiles according to duration of menopause and HRT. J Korean Acad Rehabil Med 2001;25:491-7.
7. Riggs BL, Melton LJ. The prevention and treatment of osteoporosis. N Engl J Med 1992;327:620-7.

8. Stepan JJ. Prediction of bone loss in postmenopausal women. Osteoporos Int 2000;11 Suppl 6:S45-54.

9. Kanis JA, McCloskey EV, Johansson H, Cooper C, Rizzoli R, Reginster JY, et al. European guidance for the diagnosis and management of osteoporosis in postmenopausal women. Osteoporos Int 2013;24:23-57.

10. Kanis JA, Black D, Cooper C, Dargent P, DawsonHughes B, De Laet C, et al. A new approach to the development of assessment guidelines for osteoporosis. Osteoporos Int 2002;13:527-36.

11. Vasikaran S, Eastell R, Bruyere O, Foldes AJ, Garnero P, Griesmacher A, et al. Markers of bone turnover for the prediction of fracture risk and monitoring of osteoporosis treatment: a need for international reference standards. Osteoporos Int 2011;22:391-420.

12. Kim GC, Jeong HJ, Ha HS, Lee SJ. Therapeutic effect according to estrogen dosage on combined therapy with estrogen and alendronate in postmenopausal osteoporosis. J Korean Acad Rehabil Med 2006;30:24753.

13. Vasikaran SD. Utility of biochemical markers of bone turnover and bone mineral density in management of osteoporosis. Crit Rev Clin Lab Sci 2008;45:221-58.

14. Hlaing TT, Compston JE. Biochemical markers of bone turnover: uses and limitations. Ann Clin Biochem 2014;51(Pt 2):189-202.

15. Garnero P, Sornay-Rendu E, Claustrat B, Delmas PD. Biochemical markers of bone turnover, endogenous hormones and the risk of fractures in postmenopausal women: the OFELY study. J Bone Miner Res 2000;15: 1526-36.

16. Mun KH, Kim CP, Kang BG, Lee YG. The biochemical bone marker of the pre and postmenopausal osteoporotic women. J Korean Acad Rehabil Med 1998;22:939.

17. Grampp S, Genant HK, Mathur A, Lang P, Jergas M, Takada M, et al. Comparisons of noninvasive bone mineral measurements in assessing age-related loss, fracture discrimination, and diagnostic classification. J Bone Miner Res 1997;12:697-711.

18. Ivaska KK, Lenora J, Gerdhem P, Akesson K, Vaananen HK, Obrant KJ. Serial assessment of serum bone metabolism markers identifies women with the highest rate of bone loss and osteoporosis risk. J Clin Endocri- 
nol Metab 2008;93:2622-32.

19. Delmas PD, Eastell R, Garnero P, Seibel MJ, Stepan J; Committee of Scientific Advisors of the International Osteoporosis Foundation. The use of biochemical markers of bone turnover in osteoporosis. Osteoporos Int 2000;11 Suppl 6:S2-17.

20. Rosen CJ, Chesnut CH, Mallinak NJ. The predictive value of biochemical markers of bone turnover for bone mineral density in early postmenopausal women treated with hormone replacement or calcium supplementation. J Clin Endocrinol Metab 1997;82:190410.

21. Garnero P, Hausherr E, Chapuy MC, Marcelli C, Grandjean H, Muller C, et al. Markers of bone resorption predict hip fracture in elderly women: the EPIDOS prospective study. J Bone Miner Res 1996;11:
1531-8.

22. Hochberg MC, Greenspan S, Wasnich RD, Miller P, Thompson DE, Ross PD. Changes in bone density and turnover explain the reductions in incidence of nonvertebral fractures that occur during treatment with antiresorptive agents. J Clin Endocrinol Metab 2002;87:1586-92.

23. Melton LJ, Khosla S, Atkinson EJ, O'Fallon WM, Riggs BL. Relationship of bone turnover to bone density and fractures. J Bone Miner Res 1997;12:1083-91.

24. Chavassieux P, Portero-Muzy N, Roux JP, Garnero P, Chapurlat R. Are biochemical markers of bone turnover representative of bone histomorphometry in 370 postmenopausal women? J Clin Endocrinol Metab 2015;100:4662-8. 